\title{
Mathematical Modeling and Stability Analyses of Lassa Fever Disease with the Introduction of the Carrier Compartment
}

\author{
Mary Oluwabunmi Akinade ${ }^{1 *}$, Ayodeji Sunday Afolabi ${ }^{2}$, Mark Eric Kimathi ${ }^{3}$ \\ 1. Department of Computational Mathematics, Pan African University Institute for Basic Sciences, \\ Technology and Innovation (PAUSTI), P.O. Box 62000-00200, Nairobi-Kenya
}

2. Department of Mathematical Sciences, Federal University of Technology Akure (FUTA), P.M.B. 704, Akure, Ondo State, Nigeria

3. Department of Mathematics and Statistics, Machakos University (MKsU), P.O Box 136-90100, Machakos-Kenya

*E-mail of the corresponding author: akinademary18@gmail.com

\begin{abstract}
In this paper, a new mathematical model which takes into account the human and vector populations together with their interactions during Lassa fever disease transmission was developed. This transmission process is denoted by a seven mutually exclusive compartments for the human and vector populations. The proposed model is used to introduce the incubation period of the disease, a period in which an infected individual is yet to be symptomatic but infectious however, as denoted by the carrier human compartment. This carrier compartment was critically examined for its short and long term effects on the spread and control of the disease. Local and global stability analyses of the equilibrium points of the model was carried out using the first generation matrix approach and the direct Lyapunov method respectively. These analyses showed that the disease free equilibrium point of the developed model is locally asymptotically stable but not globally asymptotically stable. It was also observed that, although, there exist a unique endemic equilibria for the disease, this equilibria however is not stable. Numerical simulations of the model were carried out by implementing the MATLAB ODE45 algorithm for solving non-stiff ordinary differential equations. The results of these simulations are the effects of the various model parameters on each compartment of the developed model. Based on the findings of this research, necessary recommendations were made for the applications of the model to an endemic area.
\end{abstract}

Keywords: Mathematical Model, Stability Analyses, Lassa Fever, Equilibrium Points, Numerical Simulation.

DOI: 10.7176/MTM/9-6-04

Publication date: June $30^{\text {th }} 2019$

\section{Introduction}

Lassa Fever (LF) is a fatal acute Viral Hemorrhagic fever caused by Lassa Virus (LASV). LASV belongs to the arena-virus family of viruses and the major host of this virus is the Mastomys (atalensis) rat specie. In addition to Person to person transmission, the other major form of transmission of the disease is from an infected animal(s) to humans. Laboratory transmission in hospitals lacking appropriate infection prevention and control measures is also common. The first occurrence of Lassa fever was in a town called "Lassa" in the present Borno state of Nigeria in 1969 and this was how the name "Lassa fever" was given to the disease. The first victim of the disease was a 65 year old female nurse who worked at the Lassa mission hospital, presently known as "Bingham University Teaching Hospital", in Borno state, Nigeria. Subsequently, the disease has been greatly spreading in Africa.

Currently, there has been an outbreak of LF in many west African countries, such as Sierra Leone, Liberia, Guinea and Nigeria, with an increasing fatal rate. The fatality rate in symptomatic, hospitalized patients ranges from $15 \%$ to $20 \%$ and as high as $90 \%$ in pregnant women [10]. There has been more outbreak of LF disease in Nigeria than in every other countries in which the disease has ever been reported. Among the 413 confirmed Lassa fever cases in Nigeria between 1st day of January and the 15th day of April 2018, 114 deaths were reported [23].

The 2018 LF outbreak in Nigeria was the largest recorded outbreak of the virus with about 1,400 suspected cases and more than 300 confirmed cases between April and June 2018 [11]. Other incidences of Lassa fever appear to be in areas of eastern sierra Leone, south-eastern Guinea, and northern Liberia. [21] reported that a total of 128 contacts, including 59 health care workers, have been line-listed and were being followed up in Liberia in June, 2018. Two deaths were reported in this outbreak. The release also disclosed that, although LF is not new to Liberia, 
it was a deadly viral disease that required an intervention. No outbreak of the fever has ever been recorded in C $\tilde{A}^{\prime} t e$ d'Ivoire, Togo and Benin, though isolated cases show evidence of viral circulation of the disease in these countries.

The most recent Lassa fever outbreak in Nigeria was between January 28 and February 3rd, 2019 with a record of 68 new confirmed cases and 14 new deaths, making it a total of 275 confirmed cases and 57 confirmed deaths in the first quarter of 2019 alone [1]. Consequently, the federal government of Nigeria declared a LF outbreak in the country as stated in a signed statement by the Director-General of the NCDC, Dr Chikwe Ihekweazu [25]. He further added that this outbreak usually occur during the Nigerian dry season, ranging from January to April of each year. The mode of transmission of LASV is through direct contact with an infected rodent or via ingestion of food which has been contaminated by the droppings, such as excreta, urine, or saliva, of an infected rodent. Most people contract LF via anything contaminated with rat urine, faeces, blood, saliva or through eating, drinking or simply handling contaminated objects in the home. LF can also be transmitted via direct contact with the blood, urine, faeces, pharyngeal secretions or other body secretions of an infected person.

The incubation period of Lassa fever, a period in which an infected person do not exhibit any symptom of the infectiousness, is a duration of 6 to 21 days from the onset of the infection. Fever, general weakness and malaise are usually the first symptoms to appear in symptomatic patients. Moreover, Lassa fever disease, at its symptomatic stage, is quite difficult to distinguish from many other diseases which cause fever, including malaria, shigellosis, typhoid fever, yellow fever and other viral hemorrhagic fevers. Moreover, in contrast with some other viral diseases, such as the Human Immune-deficiency virus (HIV), of which the Center for Disease Control and Prevention, [9], states specifically that it cannot be transmitted via water, saliva, tears, or sweat, due to its very short lifespan outside the host, LASV, however, according to the Pathogen Safety Data Sheet, [22], is stable as an aerosol and has a biological half-life, between $24^{\circ} \mathrm{C}$ and $32^{\circ} \mathrm{C}$, of 10.1 to 54.6 minutes outside host. This implies that contact with the secretions of an infected rodent or human over this period of time could still lead to the virus been transmitted.

Although, Mastomys atalensis species complex are widely distributed in sub-Sahara Africa, the ones harboring Lassa virus are the autochthonous of West Africa and that is why Lassa virus still spreads only in West Africa Countries [16]. However, there has been a report of some Lassa fever cases being "imported" into the U.S. and U.K. via travelers who acquired the disease elsewhere [13]. Presently, there is no licensed vaccine or Food and Drug Administration approved treatment against LASV [12]. However, replication-competent attenuated vaccine, one of which is Ribavirin, remains the most effective treatment against the disease especially if administered during the early stage of the infectiousness. In addition, LF disease can be prevented by practicing good hygiene, proper sanitation of the environment and the use of rodent-proof containers in storing food.

[15] investigated the prevalence of LF disease in Northern part of Edo State, Nigeria with a high rate of infection on contact persons. The results of their investigations showed that, to control the spread of the virus, the average number of new secondary infection(s) generated by a single infected individual/rodent during their infectious period, $R_{0}$, must be brought below one. Consequently, [2] developed a mathematical model for the transmission of Lassa fever with isolation of infected individuals and obtained the basic reproduction number, $R_{0}$. The analysis showed that the disease free equilibrium (DFE) is locally and globally asymptotically stable whenever the threshold quantity, $R_{0}$, is less than unity $\left(R_{0}<1\right)$. They also concluded that the endemic equilibrium point, a positive steady state solution when the disease persists in the population, of the model exists under certain condition. Similarly, [18] developed a mathematical model for the transmission of LF. This model was used to analyze the existence and stability of the DFE of LF disease. They, however, concluded that though the DFE is globally asymptotically stable (with $R_{0}<1$ ), the disease will still continue to spread. 


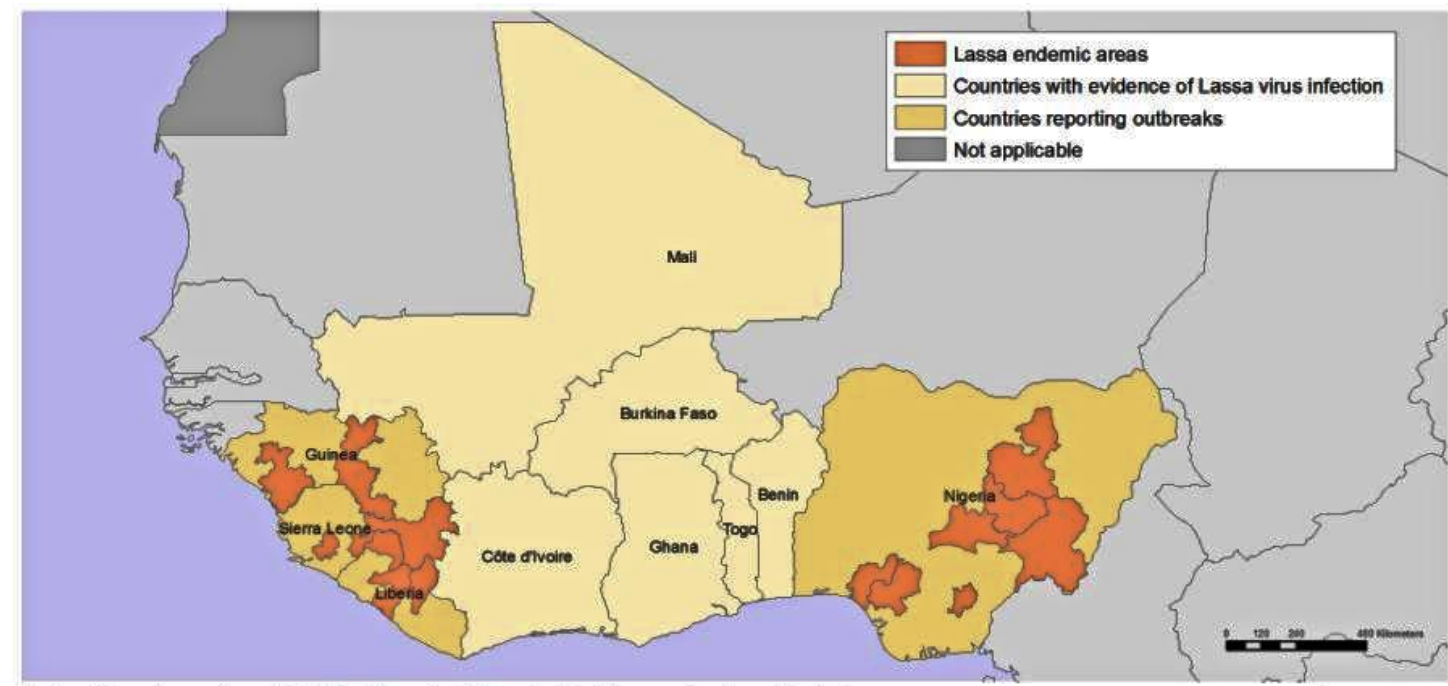

Figure 1.1: Geographic Distribution of Lassa Fever in West African affected Countries, 1969-2018 [23]

Subsequently, [17] modeled the transmission of Lassa fever virus between humans and rodents with control strategies as a six-dimensional ordinary differential equation. Stability analysis of the DFE was performed and the basic reproduction number obtained using the next generation operator approach. The existence of endemic equilibrium was further determined. The study, then, concluded that more awareness should be conducted in the affected areas so as to prevent more outbreaks of the disease. [3] modeled the control of LF disease using an SITR model. The results of their investigation showed that, though there exist an endemic equilibria for the disease, the disease can still be controlled by using appropriate control strategies. As an extension of this model, we introduced the Carrier human compartment together with new model parameters and control strategies into the previously existing model, as discussed below, in order to have a more realistic model which is closer to what is obtainable in the real life situation.

\section{Model Description and Formulation}

Mathematical modeling is one of the most important tools used in understanding the dynamics of disease transmission. In the proposed model, in order to indicate individuals with unique mutually exclusive natures, we considered five mutually exclusive compartments for the human population in relation to the disease status. These compartments are: The Susceptible $S_{h}(t)$, the Carriers $C_{h}(t)$, the Infected $I_{h}(t)$, the Treated $T_{h}(t)$ and the Recovered $R_{h}(t)$ human populations. For the rodent population, we considered two compartments namely: The Susceptible $S_{v}(t)$ and the Infected $I_{v}(t)$ vector populations.

The susceptible human compartment is made up of members who are not yet infected but stand a chance of contracting the infection if exposed to an infected individual. This is mostly because they live within a community in which the virus exists or has been previously reported. The carrier compartment consists of individuals who have the infection but do not show any clinical/noticeable symptoms even though they are infectious. The infected human compartment is made up of individuals who are with the fully blown infection with symptomatic evidence i.e they have survived the 6-21 days incubation period of the disease. The treated compartment is made up of individuals who are not only being treated but have been isolated from the other members of the community, hence are not infectious. The recovered compartment is made up of individuals who have either undergone treatment and have now fully recovered from the infection or have recovered by their own natural immunity.

We shall incorporate new model parameters such as: the rate of progression from the carrier class of the human population to the infected class to be denoted by $\sigma_{h}$, contracting rate for susceptible human population via interaction with the carriers to be denoted by $\beta_{c}$ and the rate of recovery of the carrier class by natural immunity to be denoted by $\gamma_{1}$ into the existing model of [3]. 
Table 2.1: The Model State Variables

\begin{tabular}{|c|l|}
\hline Variables & Description \\
\hline$S_{h}(t)$ & Susceptible Human Population \\
\hline$C_{h}(t)$ & Carrier Human Population \\
\hline$I_{h}(t)$ & Infected Human Population \\
\hline$T_{h}(t)$ & Treated Human Population \\
\hline$R_{h}(t)$ & Recovered Human Population \\
\hline$S_{v}(t)$ & Susceptible Vector Population \\
\hline$I_{v}(t)$ & Infected Vector Population \\
\hline
\end{tabular}

Table 2.2: The Model Parameters

\begin{tabular}{|c|l|}
\hline Parameters & Description \\
\hline$\alpha_{h}$ & Recruitment Rate into Susceptible Human Population \\
\hline$\mu_{h}$ & Natural Death Rate of Human Population \\
\hline$\omega_{h}$ & Rate of Loss of Immunity of Recovered Population \\
\hline$\beta_{c}$ & Contracting Rate for Susceptible Human Population via Interaction with the Carriers \\
\hline$\beta_{h}$ & Contracting Rate for Susceptible Human Population via Interaction with Infected Humans \\
\hline$\beta_{v}$ & Contracting rate for Susceptible Human Population via interaction with Infected Vectors \\
\hline$\delta_{h}$ & Disease-Induced Death Rate of Human Population \\
\hline$\mu_{v}$ & Natural Death Rate of Vector Population \\
\hline$\sigma_{h}$ & Rate of Progression from Carriers to Infected Human Population \\
\hline$\gamma_{2}$ & Rate of Progression from Infected Human to Treated Human Population \\
\hline$\alpha_{v}$ & Recruitment Rate into Susceptible Vector Population \\
\hline$\delta_{v}$ & Disease-Induced Death Rate of Vector Population \\
\hline$\omega_{v}$ & Rate of Progression from Susceptible Vector to Infected Vector Population \\
\hline$\gamma_{3}$ & Recovery Rate of Treated Human Population \\
\hline$\gamma_{1}$ & Rate of Recovery of the Carriers by Natural Immunity \\
\hline$\gamma_{4}$ & Rate of Recovery of Infected Human by Natural Immunity \\
\hline$\beta$ & Treatment Factor \\
\hline
\end{tabular}

Model Assumptions:

- The treated class are isolated from the remaining classes and are thus not infectious.

- Infected Rodents do not recover throughout their entire life-time.

- Treatment is given only to infected individuals.

- Infected Rodents are not given any form of Rodenticide.

- Each compartment in the model is made up of individuals or vectors with homogeneous characteristic (disease status).

- Every individual, in the studied population, who has never been infected with the virus is susceptible to the virus. 


\section{A}

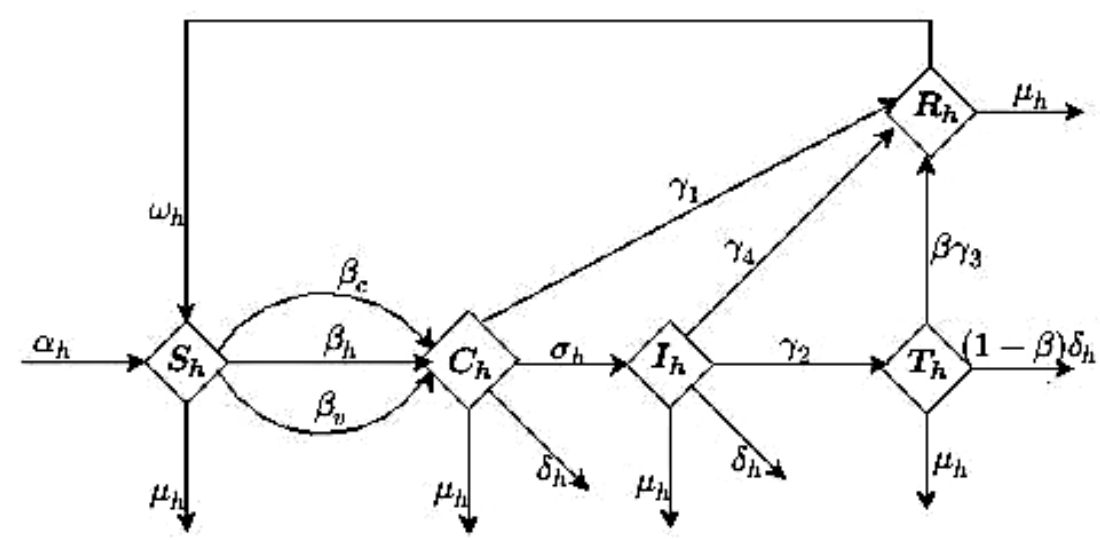

B

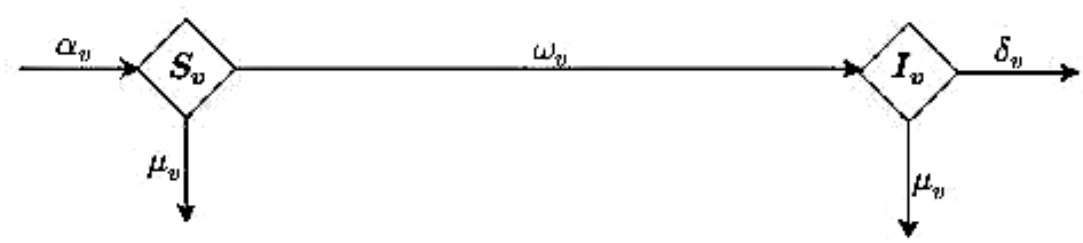

Figure 2.1: Schematic flow diagram of Lassa fever transmission in human host (A) and vector host (B).

Model Equations:

$$
\begin{aligned}
\frac{d S_{h}}{d t} & =\alpha_{h}+\omega_{h} R_{h}-\left(\beta_{c} C_{h}+\beta_{h} I_{h}+\beta_{v} I_{v}\right) S_{h}-\mu_{h} S_{h} \\
\frac{d C_{h}}{d t} & =\left(\beta_{c} C_{h}+\beta_{h} I_{h}+\beta_{v} I_{v}\right) S_{h}-\sigma_{h} C_{h}-\gamma_{1} C_{h}-\delta_{h} C_{h}-\mu_{h} C_{h} \\
\frac{d I_{h}}{d t} & =\sigma_{h} C_{h}-\gamma_{2} I_{h}-\gamma_{4} I_{h}-\delta_{h} I_{h}-\mu_{h} I_{h} \\
\frac{d T_{h}}{d t} & =\gamma_{2} I_{h}-\beta \gamma_{3} T_{h}-(1-\beta) \delta_{h} T_{h}-\mu_{h} T_{h} \\
\frac{d R_{h}}{d t} & =\gamma_{1} C_{h}+\gamma_{4} I_{h}+\beta \gamma_{3} T_{h}-\omega_{h} R_{h}-\mu_{h} R_{h} \\
\frac{d S_{v}}{d t} & =\alpha_{v}-\omega_{v} S_{v}-\mu_{v} S_{v} \\
\frac{d I_{v}}{d t} & =\omega_{v} S_{v}-\mu_{v} I_{v}-\delta_{v} I_{v}
\end{aligned}
$$

\section{The Model Analyses}

\subsection{The Invariant Region}

In this section, we shall define a region within which the solutions to the model are uniformly bounded as the set $\Omega_{1} \in R_{+}^{5}$ and $\Omega_{2} \in R_{+}^{2}$ for the human and vector populations respectively. The total human population is defined as $N_{h}(t)=S_{h}(t)+C_{h}(t)+I_{h s}(t)+T_{h}(t)+R_{h}(t)$ while that of the vector population is defined as $N_{v}(t)=$ $S_{v}(t)+I_{v}(t)$. Hence, $\frac{d N_{h}}{d t}=\frac{d S_{h}}{d t}+\frac{d C_{h}}{d t}+\frac{d I_{h}}{d t}+\frac{d T_{h}}{d t}+\frac{d R_{h}}{d t}$ while $\frac{d N_{v}}{d t}=\frac{d S_{v}}{d t}+\frac{d I_{v}}{d t}$.

From equations 2.1 to 2.7 ;

$$
\begin{gathered}
\frac{d N_{h}}{d t}=\alpha_{h}-\mu_{h} N_{h}-\delta_{h} C_{h}-\delta_{h} I_{h}-(1-\beta) \delta_{h} T_{h} \\
\frac{d N_{h}}{d t} \leq \alpha_{h}-\mu_{h} N_{h}
\end{gathered}
$$

Similarly;

$$
\begin{gathered}
\frac{d N_{v}}{d t}=\alpha_{v}-\mu_{v} N_{v}-\delta_{v} I_{v} \\
\leq \alpha_{v}-\mu_{v} N_{v}
\end{gathered}
$$

Integrating both sides of (3.1), we have;

$$
\int \frac{d N_{h}}{\alpha_{h}-\mu_{h} N_{h}} \leq \int d t
$$


where $\mathrm{C}$ is a constant of integration.

$$
\frac{-1}{\mu_{h}} \ln \left(\alpha_{h}-\mu_{h} N_{h}\right) \leq t+C
$$

Thus,

$$
\begin{gathered}
\ln \left(\alpha_{h}-\mu_{h} N_{h}\right) \geq-\left(\mu_{h} t+C\right) \\
\left(\alpha_{h}-\mu_{h} N_{h}\right) \geq A e^{-\mu_{h} t}
\end{gathered}
$$

where $\mathrm{A}$ is a constant of integration. Let $N_{h}(0)=N_{0}$, then;

Accordingly,

$$
\left(\alpha_{h}-\mu_{h} N_{0}\right) \geq A
$$

Thus, $N_{h}(t) \in\left[0, \frac{\alpha_{h}}{\mu_{h}}\right]$.

$$
\begin{gathered}
\left(\alpha_{h}-\mu_{h} N_{h}\right) \geq\left(\alpha_{h}-\mu_{h} N_{0}\right) e^{-\mu_{h} t} \\
N_{h}(t) \leq \frac{\alpha_{h}}{\mu_{h}}-\frac{\left(\alpha_{h}-\mu_{h} N_{0}\right)}{\mu_{h}} e^{-\mu_{h} t} \\
N_{h}(t) \rightarrow \frac{\alpha_{h}}{\mu_{h}} \text { as } t \rightarrow \infty
\end{gathered}
$$

Similarly, solving (3.2), we obtain; $N_{v}(t) \in\left[0, \frac{\alpha_{v}}{\mu_{v}}\right]$.

Hence, the feasible set of the solution of the model equations enter and remain in the invariant region:

$$
\Omega=\left\{\left(S_{h}, C_{h}, I_{h}, T_{h}, R_{h}\right) \in R_{+}^{5} \quad U \quad\left(S_{v}, I_{v}\right) \in R_{+}^{2}: N_{h}(t) \leq \frac{\alpha_{h}}{\mu_{h}}, N_{v}(t) \leq \frac{\alpha_{v}}{\mu_{v}}\right\}
$$

\subsection{Positivity of the Solution}

The Positivity Theorem:

Let $\Omega_{1}=\left\{\left(S_{h}, C_{h}, I_{h}, T_{h}, R_{h}\right) \in R_{+}^{5}: S_{0}>0, C_{0}>0, I_{0}>0, T_{0}>0, R_{0}>0\right\} \quad$ and $\quad \Omega_{2}=\left\{\left(S_{v}, I_{v}\right) \in R_{+}^{2}: S_{0}>\right.$ $\left.0, I_{0}>0\right\}$, then the solution of $\left\{S_{h}, C_{h}, I_{h}, T_{h}, R_{h}, S_{v}, I_{v}\right\}$ are positive for $t \geq 0$. Proof:

Considering equation 2.1 ,

$$
\begin{gathered}
\frac{d S_{h}}{d t}=\alpha_{h}+\omega_{h} R_{h}-\left(\beta_{c} C_{h}+\beta_{h} I_{h}+\beta_{v} I_{v}\right) S_{h}-\mu_{h} S_{h} \\
\geq-\left(\beta_{c} C_{h}+\beta_{h} I_{h}+\beta_{v} I_{v}+\mu_{h}\right) S_{h}
\end{gathered}
$$

thus,

$$
\begin{gathered}
\int \frac{d S_{h}}{S_{h}} \geq-\int\left(\beta_{c} C_{h}+\beta_{h} I_{h}+\beta_{v} I_{v}+\mu_{h}\right) d t \\
\ln S_{h}(t) \geq-A(t)+C \\
S_{h}(t) \geq B e^{-A(t)}
\end{gathered}
$$

Where $A(t)=\int\left(\beta_{c} C_{h}+\beta_{h} I_{h}+\beta_{v} I_{v}+\mu_{h}\right) d t$ and $C$ is a constant of integration.

At $t=0, S_{0}>0 B=e^{C} \geq 0$

Accordingly;

Similarly, considering equation 2.2 ,

$$
S_{h}(t) \geq S_{0} e^{-A(t)} \geq 0 \quad \forall \quad t \geq 0
$$

$$
\begin{gathered}
\frac{d C_{h}}{d t}=\left(\beta_{c} C_{h}+\beta_{h} I_{h}+\beta_{v} I_{v}\right) S_{h}-\sigma_{h} C_{h}-\gamma_{1} C_{h}-\delta_{h} C_{h}-\mu_{h} C_{h} \\
\geq-\left(\sigma_{h}+\gamma_{1}+\delta_{h}+\mu_{h}\right) C_{h}
\end{gathered}
$$

Thus,

$$
\begin{gathered}
\int \frac{d C_{h}}{C_{h}} \geq-\int\left(\sigma_{h}+\gamma_{1}+\delta_{h}+\mu_{h}\right) d t \\
\ln C_{h}(t) \geq-\left(\sigma_{h}+\gamma_{1}+\delta_{h}+\mu_{h}\right) t+D
\end{gathered}
$$

Where D is a constant of integration.

Where $A=\left(\sigma_{h}+\gamma_{1}+\delta_{h}+\mu_{h}\right) \geq 0$

$$
C_{h}(t) \geq B e^{-A t}
$$

At $t=0, C_{0}>0 B=e^{D} \geq 0$ 
Accordingly;

Next, considering equation 2.3,

$$
C_{h}(t) \geq C_{0} e^{-A t} \geq 0 \quad \forall \quad t \geq 0
$$

$$
\begin{aligned}
\frac{d I_{h}}{d t}= & \sigma_{h} C_{h}-\gamma_{2} I_{h}-\gamma_{4} I_{h}-\delta_{h} I_{h}-\mu_{h} I_{h} \\
& \geq-\left(\gamma_{2}+\gamma_{4}+\delta_{h}+\mu_{h}\right) I_{h}
\end{aligned}
$$

Hence,

$$
I_{h}(t) \geq I_{0} e^{-A t} \geq 0 \quad \forall \quad t \geq 0
$$

Where $A=\left(\gamma_{2}+\gamma_{4}+\delta_{h}+\mu_{h}\right) \geq 0$

In the same way, considering equation 2.4 we have;

Where $A=\left(\beta \gamma_{3}+(1-\beta) \delta_{h}+\mu_{h}\right) \geq 0$

$$
T_{h}(t) \geq T_{0} e^{-A t} \geq 0 \quad \forall \quad t \geq 0
$$

and considering equation 2.5 , we have;

where $A=\left(\omega_{h}+\mu_{h}\right) \geq 0$

$$
R_{h}(t) \geq R_{0} e^{-A t} \geq 0 \quad \forall \quad t \geq 0
$$

Next, considering equation 2.6, we obtain;

where $A=\left(\omega_{v}+\mu_{v}\right) \geq 0$

$$
S_{v}(t) \geq S_{0} e^{-A t} \geq 0 \quad \forall \quad t \geq 0
$$

and lastly, considering equation 2.7 , we obtain;

$$
I_{v}(t) \geq I_{0} e^{-A t} \geq 0 \quad \forall \quad t \geq 0
$$

where $A=\left(\mu_{v}+\delta_{v}\right) \geq 0$

This completes the proof of the theorem.

\subsection{The Equilibrium Points}

\subsubsection{The Disease Free Equilibrium Point (DFE):}

The DFE of the model is defined as $\left(S_{h}^{*}(t), 0,0,0,0, S_{v}^{*}(t), 0\right)$ satisfying;

$\frac{d S_{h}}{d t}=\frac{d C_{h}}{d t}=\frac{d I_{h}}{d t}=\frac{d T_{h}}{d t}=\frac{d R_{h}}{d t}=0$ and $\frac{d S_{v}}{d t}=\frac{d I_{v}}{d t}=0$ and $\frac{d S_{v}}{d t}=\frac{d I_{v}}{d t}=0$

By equating equations (2.1) to (2.7) to 0 and substituting $C_{h}=I_{h}=T_{h}=R_{h}=I_{v}=0$, We obtain $S_{h}^{*}=\frac{\alpha_{h}}{\mu_{h}}$. Accordingly, the DFE is obtained as:

$$
E_{0}=\left(\frac{\alpha_{h}}{\mu_{h}}, 0,0,0,0\right)
$$

\subsubsection{The Endemic Equilibrium Point}

The EEP of the model is defined as $\left(S_{h}^{*}(t), C_{h}^{*}(t), I_{h}^{*}(t), T_{h}^{*}(t), R_{h}^{*}(t), S_{v}^{*}(t), I_{v}^{*}(t)\right)$ satisfying;

$\frac{d S_{h}}{d t}=\frac{d C_{h}}{d t}=\frac{d I_{h}}{d t}=\frac{d T_{h}}{d t}=\frac{d R_{h}}{d t}=0$ and $\frac{d S_{v}}{d t}=\frac{d I_{v}}{d t}=0$

Solving $\frac{d S_{v}}{d t}=\frac{d I_{v}}{d t}=0$, we obtain;

$\alpha_{v}-\left(\omega_{v}+\mu_{v}\right) S_{v}=0$ and $\omega_{v} S_{v}-\left(\mu_{v}+\delta_{v}\right) I_{v}=0$

which yields:

and

$$
S_{v}^{*}=\frac{\alpha_{v}}{\left(\omega_{v}+\mu_{v}\right)}
$$

Next, equating equation (2.3) to 0 , we obtain:

$$
I_{v}^{*}=\frac{\omega_{v} \alpha_{v}}{\left(\mu_{v}+\delta_{v}\right)\left(\omega_{v}+\mu_{v}\right)}
$$

where $A=\gamma_{2}+\gamma_{4}+\delta_{h}+\mu_{h}$

$$
I_{h}^{*}=\frac{\sigma_{h} C_{h}^{*}}{A}
$$

Similarly, equating equation (2.4) to 0 , we obtain:

$$
\begin{aligned}
& T_{h}^{*}=\frac{\gamma_{2} I_{h}^{*}}{B} \\
& =\frac{\gamma_{2} \sigma_{h} C_{h}^{*}}{A B}
\end{aligned}
$$


where $B=\beta \gamma_{3}+(1-\beta) \delta_{h}+\mu_{h}$

Similarly, equating equation (2.5) to 0 , we obtain:

$$
\begin{gathered}
R_{h}^{*}=\frac{\gamma_{1} C_{h}^{*}+\gamma_{4} I_{h}^{*}+\beta \gamma_{3} T_{H}^{*}}{C} \\
=\frac{D C_{h}^{*}}{A B C}
\end{gathered}
$$

where $C=\omega_{h}+\mu_{h} \quad$ and $\quad D=A B \gamma_{1}+B \gamma_{4} \sigma_{h}+\beta \gamma_{2} \gamma_{3} \sigma_{h}$

Now, adding equations (2.1) to (2.5) at $\frac{d S_{h}}{d t}=\frac{d C_{h}}{d t}=\frac{d I_{h}}{d t}=\frac{d T_{h}}{d t}=\frac{d R_{h}}{d t}=0$, we have;

which yields:

$$
\alpha_{h}-\mu_{h} S_{h}^{*}-\left(\delta_{h}+\mu_{h}\right) C_{h}^{*}-\left(\delta_{h}+\mu_{h}\right) I_{h}^{*}-\left((1-\beta) \delta_{h}+\mu_{h}\right) T_{h}^{*}-\mu_{h} R_{h}^{*}=0
$$

where $b=(1-\beta)$

$$
\alpha_{h}-\mu_{h} S_{h}^{*}-\left(\delta_{h}+\mu_{h}\right) C_{h}^{*}-\left(\delta_{h}+\mu_{h}\right) \frac{\sigma_{h} C_{h}^{*}}{A}-\left(b \delta_{h}+\mu_{h}\right) \frac{\gamma_{2} \sigma_{h} C_{h}^{*}}{A B}-\mu_{h} \frac{D C_{h}^{*}}{A B C}=0
$$

Accordingly;

$$
\alpha_{h}-\mu_{h} S_{h}^{*}-\frac{E C_{h}^{*}}{A B C}=0
$$

where $E=D \mu_{h}+\left(b \delta_{h}+\mu_{h}\right) \gamma_{2} \sigma_{h} C+\left(\delta_{h}+\mu_{h}\right) \sigma_{h} B C+\left(\delta_{h}+\mu_{h}\right) A B C$

Thus:

$$
C_{h}^{*}=\frac{A B C}{E}\left(\alpha_{h}-\mu_{h} S_{h}^{*}\right)
$$

Substituting equation (3.18) into equations (3.14) to (3.16), we obtain:

$$
\begin{aligned}
& I_{h}^{*}=\frac{\sigma_{h} B C}{E}\left(\alpha_{h}-\mu_{h} S_{h}^{*}\right) \\
& T_{h}^{*}=\frac{\gamma_{2} \sigma_{h} C}{E}\left(\alpha_{h}-\mu_{h} S_{h}^{*}\right) \\
& R_{h}^{*}=\frac{D}{E}\left(\alpha_{h}-\mu_{h} S_{h}^{*}\right)
\end{aligned}
$$

Clearly, all the state variables, $\left(S_{h}^{*}, C_{h}^{*}, I_{h}^{*}, T_{h}^{*}, R_{h}^{*}, S_{v}^{*}, I_{v}^{*}\right)$, are strictly positive since $\left(\alpha_{h}-\mu_{h} S_{h}^{*}\right) \geq 0$ and in fact $\left(\alpha_{h}-\mu_{h} S_{h}^{*}\right)=0$ only at the DFE.

Hence there exist an EEP, $E^{*}=\left(S_{h}^{*}(t), C_{h}^{*}(t), I_{h}^{*}(t), T_{h}^{*}(t), R_{h}^{*}(t), S_{v}^{*}(t), I_{v}^{*}(t)\right)$.

\subsection{Local Asymptotic Stability Analysis}

\section{The Basic Reproduction Number:}

According to the principle of next generation matrix, the basic reproduction number is the spectral radius of the next generation matrix $F V^{-1}$ of the system (2.1) to (2.7). Where:

Accordingly;

$$
\begin{gathered}
f_{i}-v_{i}=\left(\begin{array}{l}
C_{h^{\prime}} \\
I_{h^{\prime}} \\
T_{h^{\prime}}
\end{array}\right) \\
=\left(\begin{array}{l}
\left(\beta_{c} C_{h}+\beta_{h} I_{h}+\beta_{v} I_{v}\right) S_{h}-\left(\sigma_{h}+\gamma_{1}+\delta_{h}+\mu_{h}\right) C_{h} \\
\sigma_{h} C_{h}-\left(\gamma_{2}+\gamma_{4}+\delta_{h}+\mu_{h}\right) I_{h} \\
\gamma_{2} I_{h}-\left(\beta \gamma_{3}+(1-\beta) \delta_{h}+\mu_{h}\right) T_{h}
\end{array}\right)
\end{gathered}
$$

And;

$$
f_{i}=\left(\begin{array}{c}
\left(\beta_{c} C_{h}+\beta_{h} I_{h}+\beta_{v} I_{v}\right) S_{h} \\
0 \\
0
\end{array}\right)
$$

$$
v_{i}=\left(\begin{array}{l}
\left(\sigma_{h}+\gamma_{1}+\delta_{h}+\mu_{h}\right) C_{h} \\
\left(\gamma_{2}+\gamma_{4}+\delta_{h}+\mu_{h}\right) I_{h}-\sigma_{h} C_{h} \\
\left(\beta \gamma_{3}+(1-\beta) \delta_{h}+\mu_{h}\right) T_{h}-\gamma_{2} I_{h}
\end{array}\right)
$$

where $f_{i}$ is the rate of appearance of new infection(s) in compartment $\mathrm{i}$ and $v_{i}$ represents the rate of transfer of individuals into compartment $\mathrm{i}$, with $i \in[1,3]$.

The matrix $\mathrm{F}$ and $\mathrm{V}$ are obtained as follows: 
and;

$$
\begin{gathered}
F=\left(\begin{array}{lll}
\frac{\partial f_{1}}{\partial c_{h}} & \frac{\partial f_{1}}{\partial I_{h}} & \frac{\partial f_{1}}{\partial T_{h}} \\
\frac{\partial f_{2}}{\partial C_{h}} & \frac{\partial f_{2}}{\partial I_{h}} & \frac{\partial f_{2}}{\partial T_{h}} \\
\frac{\partial f_{3}}{\partial C_{h}} & \frac{\partial f_{3}}{\partial I_{h}} & \frac{\partial f_{3}}{\partial T_{h}}
\end{array}\right) \\
=\left(\begin{array}{ccc}
\beta_{c} S_{h} & \beta_{h} S_{h} & 0 \\
0 & 0 & 0 \\
0 & 0 & 0
\end{array}\right)
\end{gathered}
$$

$$
V=\left(\begin{array}{lll}
\frac{\partial v_{1}}{\partial C_{h}} & \frac{\partial v_{1}}{\partial I_{h}} & \frac{\partial v_{1}}{\partial T_{h}} \\
\frac{\partial v_{2}}{\partial c_{h}} & \frac{\partial v_{2}}{\partial I_{h}} & \frac{\partial v_{2}}{\partial T_{h}} \\
\frac{\partial v_{3}}{\partial c_{h}} & \frac{\partial v_{3}}{\partial I_{h}} & \frac{\partial v_{3}}{\partial T_{h}}
\end{array}\right)
$$

$$
=\left(\begin{array}{ccc}
\left(\sigma_{h}+\gamma_{1}+\delta_{h}+\mu_{h}\right) & 0 & 0 \\
-\sigma_{h} & \left(\gamma_{2}+\gamma_{4}+\delta_{h}+\mu_{h}\right) & 0 \\
0 & -\gamma_{2} & \left(\beta \gamma_{3}+(1-\beta) \delta_{h}+\mu_{h}\right)
\end{array}\right)
$$

Lastly, $V^{-1}$

$$
\left(\begin{array}{ccc}
\frac{1}{\delta_{h}+\gamma_{1}+\mu_{h}+\sigma_{h}} & 0 & 0 \\
\frac{\sigma_{h}}{\left(\delta_{h}+\gamma_{1}+\mu_{h}+\sigma_{h}\right)\left(\delta_{h}+\gamma_{2}+\gamma_{4}+\mu_{h}\right)} & \frac{1}{\gamma_{h}+\gamma_{2}+\gamma_{4}+\mu_{h}} & 0 \\
-\frac{\gamma_{2} \sigma_{h}}{\left((\beta-1) \delta_{h}-\beta \gamma_{3}-\mu_{h}\right)\left(\delta_{h}+\gamma_{1}+\mu_{h}+\sigma_{h}\right)\left(\delta_{h}+\gamma_{2}+\gamma_{4}+\mu_{h}\right)} & -\frac{\gamma_{2}}{\left((\beta-1) \delta_{h}-\beta \gamma_{3}-\mu_{h}\right)\left(\delta_{h}+\gamma_{2}+\gamma_{4}+\mu_{h}\right)} & -\frac{1}{(\beta-1) \delta_{h}-\beta \gamma_{3}-\mu_{h}}
\end{array}\right)
$$

Thus, the next generation matrix:

$$
\begin{aligned}
& G=F V^{-1} \\
& =\left(\begin{array}{ccc}
\frac{s_{h} \beta_{c}}{\delta_{h}+\gamma_{1}+\mu_{h}+\sigma_{h}}+\frac{s_{h} \beta_{h} \sigma_{h}}{\left(\delta_{h}+\gamma_{1}+\mu_{h}+\sigma_{h}\right)\left(\delta_{h}+\gamma_{2}+\gamma_{4}+\mu_{h}\right)} & \frac{s_{h} \beta_{h}}{\delta_{h}+\gamma_{2}+\gamma_{4}+\mu_{h}} & 0 \\
0 & 0 & 0 \\
0 & 0 & 0
\end{array}\right)
\end{aligned}
$$

The eigenvalues of the Matrix, G, are:

$$
\begin{gathered}
{\left[\frac{S_{h} \beta_{c} \delta_{h}+S_{h} \beta_{c} \gamma_{2}+S_{h} \beta_{c} \gamma_{4}+S_{h} \beta_{c} \mu_{h}+S_{h} \beta_{h} \sigma_{h}}{\delta_{h}^{2}+\delta_{h} \gamma_{1}+\left(\delta_{h}+\gamma_{1}\right) \gamma_{2}+\left(\delta_{h}+\gamma_{1}\right) \gamma_{4}+\left(2 \delta_{h}+\gamma_{1}+\gamma_{2}+\gamma_{4}\right) \mu_{h}+\mu_{h}^{2}+\left(\delta_{h}+\gamma_{2}+\gamma_{4}+\mu_{h}\right) \sigma_{h}}, 0,0\right]} \\
=\left[\frac{s_{h}^{*} \beta_{c}}{\left(\delta_{h}+\gamma_{1}+\mu_{h}+\sigma_{h}\right)}+\frac{s_{h}^{*} \beta_{h} \sigma_{h}}{\left(\delta_{h}+\gamma_{1}+\mu_{h}+\sigma_{h}\right)\left(\delta_{h}+\gamma_{2}+\gamma_{4}+\mu_{h}\right)}, 0,0\right]
\end{gathered}
$$

Hence, the spectral radius of $\mathrm{G}$ is;

$$
\begin{aligned}
& R_{0}=R_{1}+R_{2}=\frac{\alpha_{h} \beta_{c}}{\mu_{h}\left(\delta_{h}+\gamma_{1}+\mu_{h}+\sigma_{h}\right)}+\frac{\alpha_{h} \beta_{h} \sigma_{h}}{\mu_{h}\left(\delta_{h}+\gamma_{2}+\gamma_{4}+\mu_{h}\right)\left(\delta_{h}+\gamma_{1}+\mu_{h}+\sigma_{h}\right)} \\
& =\frac{\left(\beta_{c} \delta_{h}+\beta_{c} \gamma_{2}+\beta_{c} \gamma_{4}+\beta_{c} \mu_{h}+\beta_{h} \sigma_{h}\right) S_{h}^{*}}{\delta_{h}^{2}+\delta_{h} \gamma_{1}+\left(\delta_{h}+\gamma_{1}\right) \gamma_{2}+\left(\delta_{h}+\gamma_{1}\right) \gamma_{4}+\left(2 \delta_{h}+\gamma_{1}+\gamma_{2}+\gamma_{4}\right) \mu_{h}+\mu_{h}^{2}+\left(\delta_{h}+\gamma_{2}+\gamma_{4}+\mu_{h}\right) \sigma_{h}}
\end{aligned}
$$

Accordingly, by substituting the values in table (3.1) below the basic reproduction number is obtained as;

$$
R_{0}=0.46252994
$$


Table 3.1: Table of Values

\begin{tabular}{|c|l|l|}
\hline Parameters & Values & Sources \\
\hline$\alpha_{h}$ & 0.0915 & Estimate \\
\hline$\mu_{h}$ & 0.0000548 & {$[3]$} \\
\hline$\omega_{h}$ & 0.25 & Estimate \\
\hline$\beta_{c}$ & 0.000062 & {$[15]$} \\
\hline$\beta_{h}$ & 0.00012 & Estimate \\
\hline$\beta_{v}$ & 0.005 & {$[3]$} \\
\hline$\delta_{h}$ & 0.001 & {$[9]$} \\
\hline$\mu_{v}$ & 0.000167 & Estimate \\
\hline$\sigma_{h}$ & 0.50 & {$[17]$} \\
\hline$\gamma_{2}$ & 0.70 & Estimate \\
\hline$\alpha_{v}$ & 0.70 & {$[5]$} \\
\hline$\delta_{v}$ & 0.05 & Estimate \\
\hline$\omega_{v}$ & 0.02 & Estimate \\
\hline$\gamma_{3}$ & 0.50 & {$[17]$} \\
\hline$\gamma_{1}$ & 0.0315 & Estimate \\
\hline$\gamma_{4}$ & 0.0005 & Estimate \\
\hline$\beta$ & 0.45 & Estimate \\
\hline
\end{tabular}

The Principle of Next Generation Matrix:

The DFE of an infectious disease is LAS if the basic reproduction number, $R_{0}<1$ and the EEP, if it exist, is unstable.

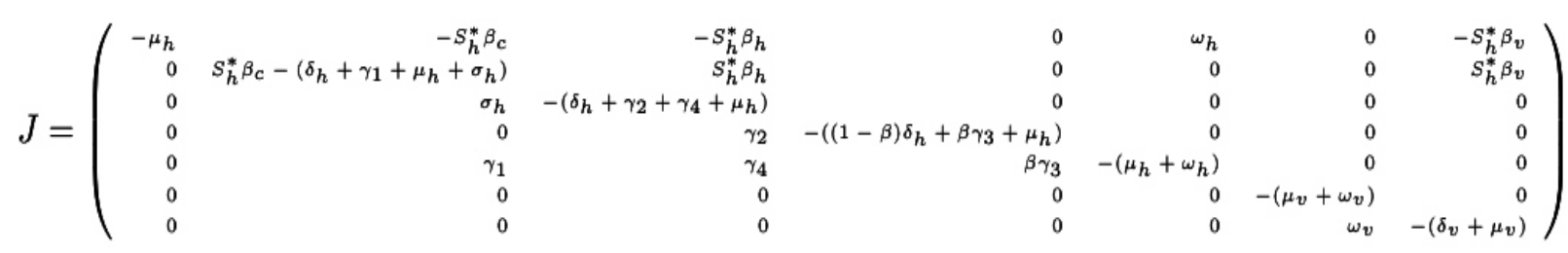

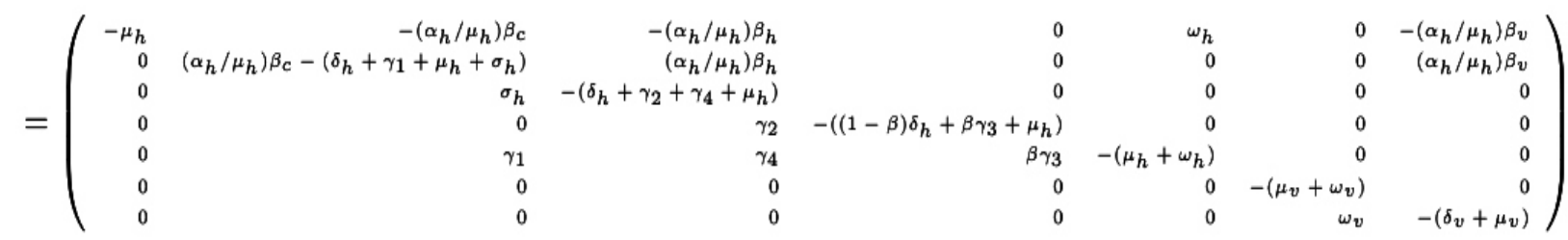

The eigenvalues of the Jacobian matrix, J, are :

$$
\lambda_{1,2}=\left(\frac{\alpha_{h} \beta_{c}-\left(2 \delta_{h}+\gamma_{1}+\gamma_{2}+\gamma_{4}\right) \mu_{h}-2 \mu_{h}^{2}-\mu_{h} \sigma_{h} \pm \sqrt{Z}}{2 \mu_{h}}\right)
$$

$\lambda_{3}=-\left(\mu_{v}+\omega_{v}\right), \lambda_{4}=-\left(\mu_{h}+\omega_{h}\right), \lambda_{5}=-\left(\delta_{v}+\mu_{v}\right), \lambda_{6}=-(1-\beta) \delta_{h}+\beta \gamma_{3}+\mu_{h}$ and $\lambda_{7}=-\mu_{h}$.

where;

$$
\begin{gathered}
Z=\alpha_{h}^{2} \beta_{c}^{2}+\mu_{h}^{2} \sigma_{h}^{2}+\left(\gamma_{1}^{2}-2 \gamma_{1} \gamma_{2}+\gamma_{2}^{2}-2\left(\gamma_{1}-\gamma_{2}\right) \gamma_{4}+\gamma_{4}^{2}\right) \mu_{h}^{2}-2\left(\alpha_{h} \beta_{c} \gamma_{1}-\alpha_{h} \beta_{c} \gamma_{2}-\alpha_{h} \beta_{c} \gamma_{4}\right) \mu_{h} \\
+2\left(\left(\gamma_{1}-\gamma_{2}-\gamma_{4}\right) \mu_{h}^{2}-\left(\alpha_{h} \beta_{c}-2 \alpha_{h} \beta_{h}\right) \mu_{h}\right) \sigma_{h}
\end{gathered}
$$

Clearly, all the eigenvalues of the Jacobian matrix are strictly negative provided:

$\left(\frac{\alpha_{h} \beta_{c}-\left(2 \delta_{h}+\gamma_{1}+\gamma_{2}+\gamma_{4}\right) \mu_{h}-2 \mu_{h}-\mu_{h} \sigma_{h}+\sqrt{Z}}{2}\right)<0$. Thus, it follows that if $R_{0}$ as defined in equation (3.29) is less than unity, then the DFE ${ }^{2} h_{\text {is }}$ locally asymptotically stable.

Accordingly, since the threshold parameter $R_{0}=0.46252994<1$, then the DFE is LAS and the EEP is unstable, hence the disease cannot invade the population. However, the existence of the Endemic equilibria implies that the disease may persist in the population. 


\subsection{Global Asymptotic Stability Analysis}

In order to examine the DFE for global stability, we shall employ the procedure implemented by [8]. We shall denote the Lassa fever model by:

$$
\left(\begin{array}{l}
\frac{d X}{d t}=F(X, Y) \\
\frac{d Y}{d t}=G(X, Y)
\end{array}\right.
$$

where $X=\left(S_{h}, R_{h}, S_{v}\right)$ denotes the uninfected population and $Y=\left(C_{h}, I_{h}, T_{h}, I_{v}\right)$ denotes the Infected population.

The point $E_{0}=\left(X^{*}, 0\right)$ is said to be globally asymptotically stable if $R_{0}<1$ and in addition the following two conditions hold:

For C1:

C1: For $\frac{d X}{d t}=F(X, 0), E_{0}$ is globally asymptotically stable.

C2: $G(X, Y)=A Y-G^{*}(X, Y), G^{*}(X, Y) \geq 0$ for $(X, Y) \in \Omega$

$$
F(X, 0)=\left(\begin{array}{l}
\alpha_{h}+\omega_{h} R_{h}-\mu_{h} S_{h} \\
-\left(\omega_{h}+\mu_{h}\right) R_{h} \\
\alpha_{v}-\left(\omega_{v}+\mu_{v}\right) S_{v}
\end{array}\right.
$$

Clearly, $E_{0}=\left(\frac{\alpha_{h}}{\mu_{h}}, 0,0,0,0, \frac{\alpha_{v}}{\mu_{v}}, 0\right)$ is globally asymptotically stable for $\frac{d X}{d t}=F(X, 0)$. This can be verified as shown below: By solving equation (3.32) using the method of integrating factor, we have:

Thus, $S_{h}=\frac{\alpha_{h}}{\mu_{h}}-\frac{1}{e^{\mu_{h} t}} \int \omega_{h} R_{h} e^{\mu_{h} t} d t$

$$
\begin{gathered}
\frac{d S_{h}}{d t}=\alpha_{h}+\omega_{h} R_{h}-\mu_{h} S_{h} \\
I . F=e^{\int \mu_{h} d t} \\
\left(\frac{d S_{h}}{d t}+\mu_{h} S_{h}\right) e^{\mu_{h} t}=\left(\alpha_{h}-\omega_{h} R_{h}\right) e^{\mu_{h} t} \\
\frac{d}{d t}\left(S_{h} e^{\mu_{h} t}\right)=\left(\alpha_{h}-\omega_{h} R_{h}\right) e^{\mu_{h} t}
\end{gathered}
$$

accordingly, $S_{h}(t) \rightarrow \frac{\alpha_{h}}{\mu_{h}}$ as $t \rightarrow \infty$

Similarly, $S_{v}(t) \rightarrow \frac{\alpha_{v}}{\mu_{v}}$ as $t \rightarrow \infty$

which implies the global convergence of 3.32 in $\Omega$.

For C2:

$$
\begin{aligned}
& G(X, Y)=\left(\begin{array}{l}
\left(\beta_{c} C_{h}+\beta_{h} I_{h}+\beta_{v} I_{v}\right) S_{h}-\left(\sigma_{h}+\gamma_{1}+\delta_{h}+\mu_{h}\right) C_{h} \\
\sigma_{h} C_{h}-\left(\gamma_{2}+\gamma_{4}+\delta_{h}+\mu_{h}\right) I_{h} \\
\gamma_{2} I_{h}-\left(\beta \gamma_{3}+b \delta_{h}+\mu_{h}\right) T_{h} \\
\omega_{v} S_{v}-\left(\mu_{v}+\delta_{v}\right) I_{v}
\end{array}\right. \\
& =A Y-G^{*}(X, Y)
\end{aligned}
$$

Where:

$$
\left(\begin{array}{cccc}
-\left(\sigma_{h}+\gamma_{1}+\delta_{h}+\mu_{h}\right) & 0 & 0 & 0 \\
\sigma_{h} & -\left(\gamma_{2}+\gamma_{4}+\delta_{h}+\mu_{h}\right) & 0 & 0 \\
0 & \gamma_{2} & -\left(\beta \gamma_{3}+b \delta_{h}+\mu_{h}\right) & 0 \\
0 & 0 & 0 & -\left(\mu_{v}+\delta_{v}\right)
\end{array}\right)
$$

and;

$$
G^{*}(X, Y)=\left[\begin{array}{l}
-\left(\beta_{c} C_{h}+\beta_{h} I_{h}+\beta_{v} I_{v}\right) S_{h} \\
0 \\
0 \\
-\omega_{v} S_{v}
\end{array}\right]
$$

Clearly, $G^{*}(X, Y) \leq 0$. Hence, condition 2 is not satisfied. Thus, $E_{0}=\left(X^{*}, 0\right)$ may not be GAS for $R_{0}<1$. 


\section{Numerical Simulations and Discussion of Results}

In order to solve the model equations numerically, we implemented the MATLAB ODE45 algorithm for the developed model, and plotted the graphs of each model compartment against time, with time ranging from 0 to 150 days, we obtained the following results:

\subsection{Susceptible Human Population}

Figure 4.1 indicates that the susceptible human population decreases rapidly within the first few days due to the awareness/sensitivity of the affected population towards the infection and also because of the progression from the susceptible class to the carrier class. However, after these few days, an inflexion point is reached and then the population begins to increase steadily for the remaining 100 days due to the loss of immunity of the recovered human population. This result is in agreement with the results of [20] and [6]. It can be observed that an increase in the contracting rate of the susceptible class via contact with the carrier class, $\beta_{c}$, leads to a decrease in the population of this compartment.

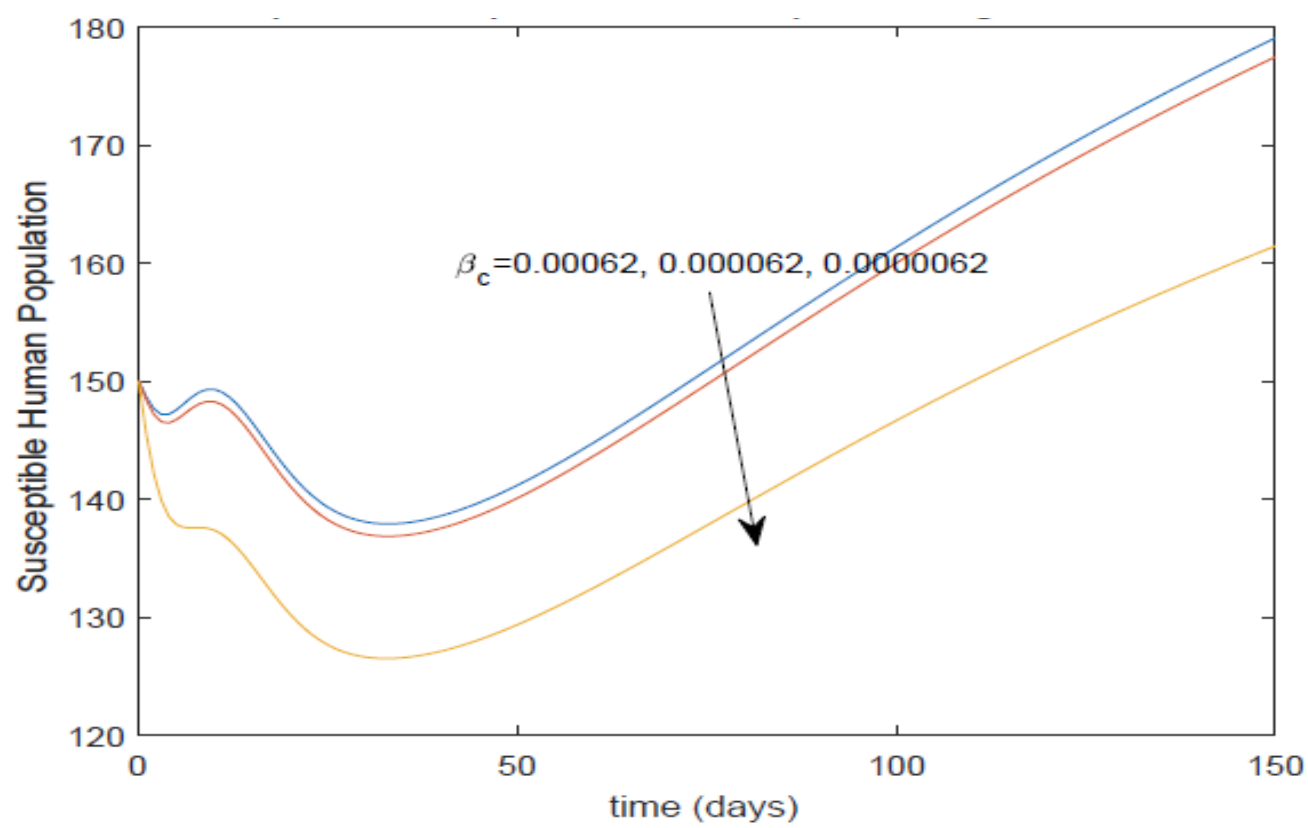

Figure 4.1: Graph of the Susceptible Human Population against Time varying $\beta_{c}$

\subsection{Carrier Human Population}

In figure 4.2 to 4.4 , the introduced carrier compartment reduces drastically in size from 40 to 19 during the first 5 days. This is unsurprisingly due to the progression of the carriers to the infected compartment since the incubation period of the disease is relatively short (6 to 21 days) and also due to the disease-induced death of the class. Thereafter, the compartment begins to increase in its population from 19 to 25 within a duration of 20 days due to the inflow from the susceptible compartment. Subsequently, the population was maintained at 25 due to the balance achieved in the inflow and outflow of humans in this compartment. It can be observed that an increase in the rate of recovery of the Carrier class, $\gamma_{1}$ and the progression rate from the carrier class to the infected class, $\sigma_{h}$, lead to a decrease in the population of the carrier class. Meanwhile an increase in the contracting rate of the susceptible class via contact with the carrier class, $\beta_{c}$, leads to an increase in the population of the carriers. 


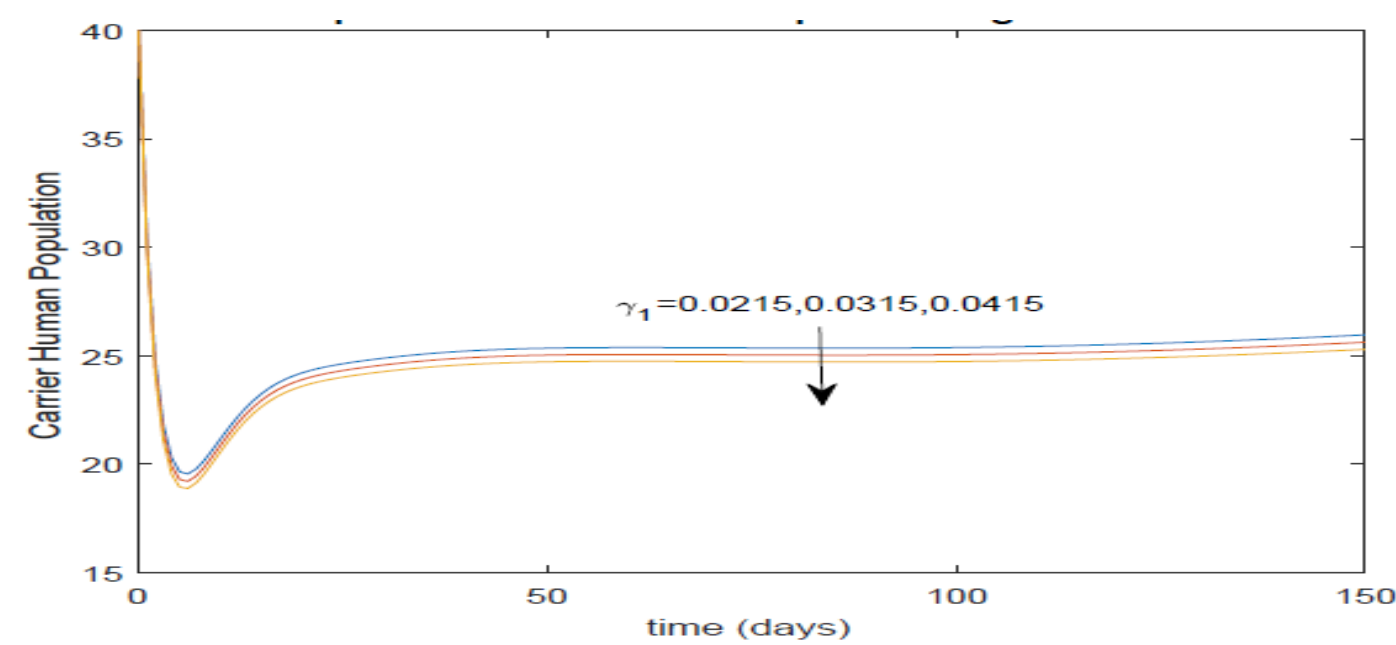

Figure 4.2: Graph of the Carrier Human Population against Time varying $\gamma_{1}$

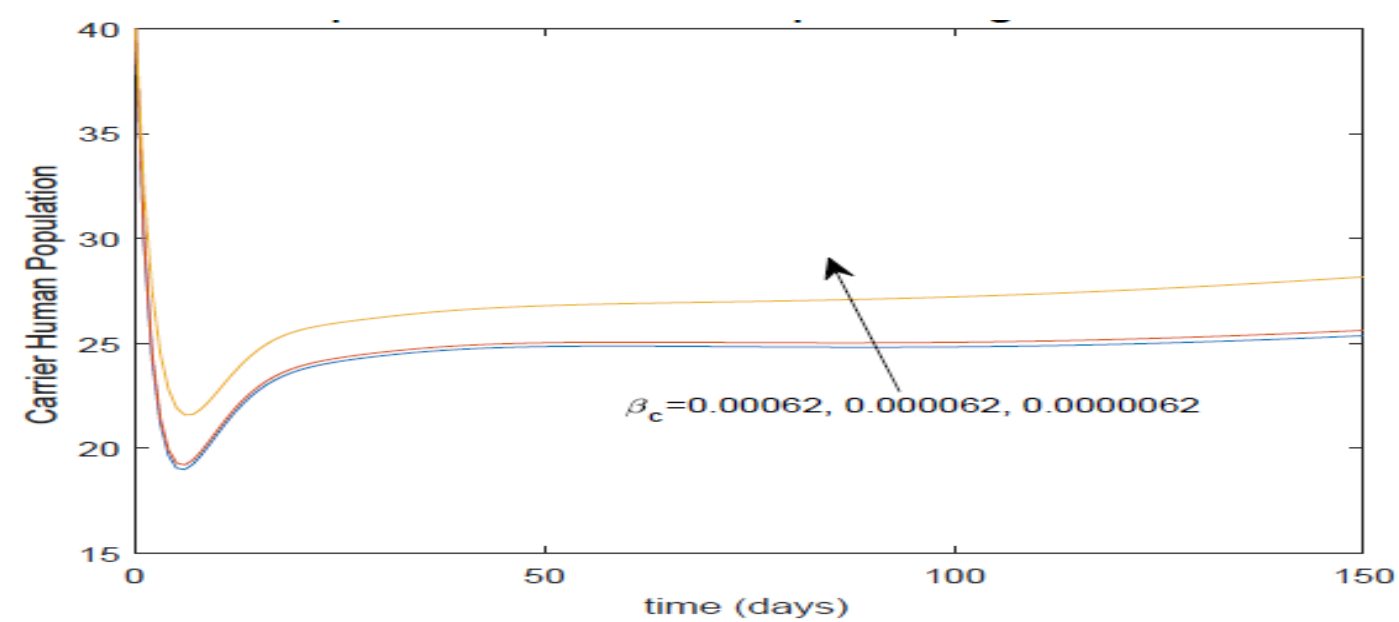

Figure 4.3: Graph of the Carrier Human Population against Time varying varying $\beta$

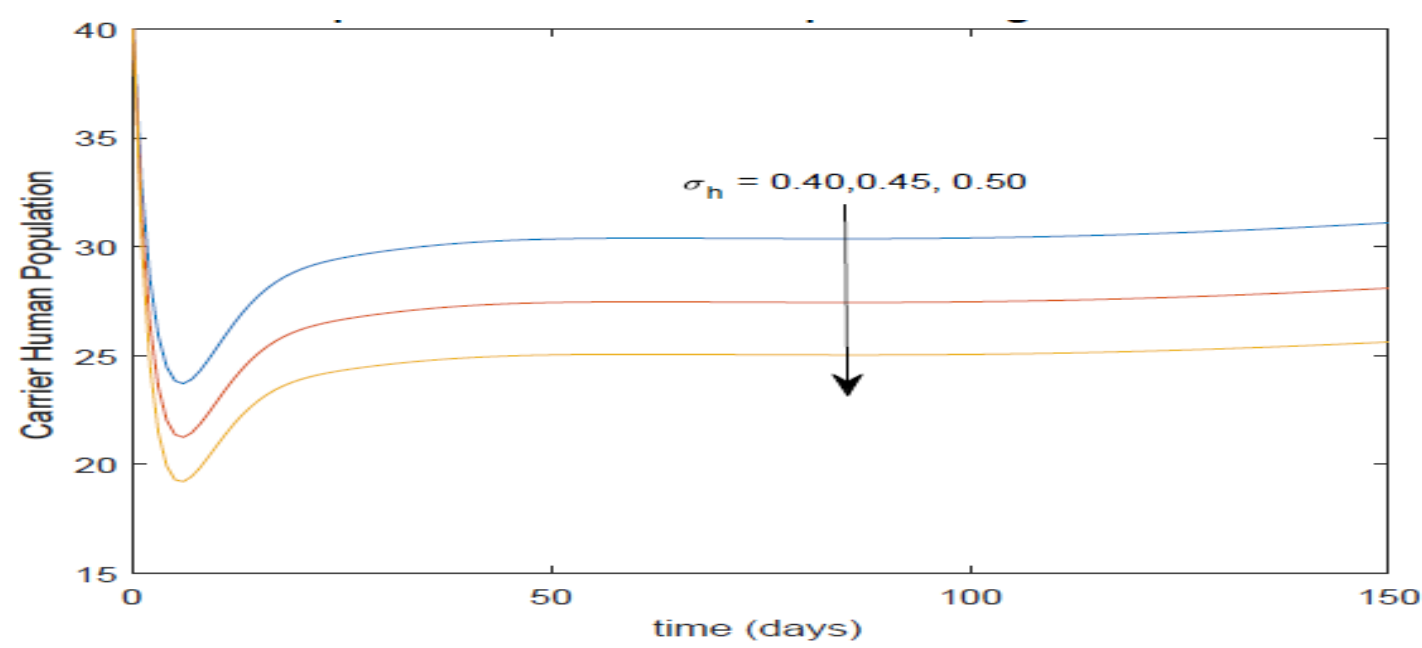

Figure 4.4: Graph of the Carrier Human Population against Time varying varying $\sigma_{h}$

\subsection{Infected Human Population}

From figure 4.5, the infected human population experiences a rapid decrease within the first 5 days. This decrease can be ascribed to the availability of treatment for the infected compartment since they are symptomatic and thus easily diagnosable compared to the carriers who are without symptom. After this period, however, there is a short increase in the population of this compartment because of the progression from the carrier compartment. Thereafter, the population of this compartment is maintained at a stable value of 17 because of the balance in the inflow and out flow of individuals within this population. This result is in slight contrast with those in previous 
literatures. According to [3] and [20], the infected human compartment experiences a rapid increase between time 0 and 5. This increase was ascribed to the progression from the susceptible human population to the infected human population. This, however, is not true in our case as there do not exist a progression into the infected human compartment directly from the susceptible human compartment. It can be observed that an increase in the progression rate from the carrier class to the infected class, $\sigma_{h}$, lead to an increase in the population of the infected class.

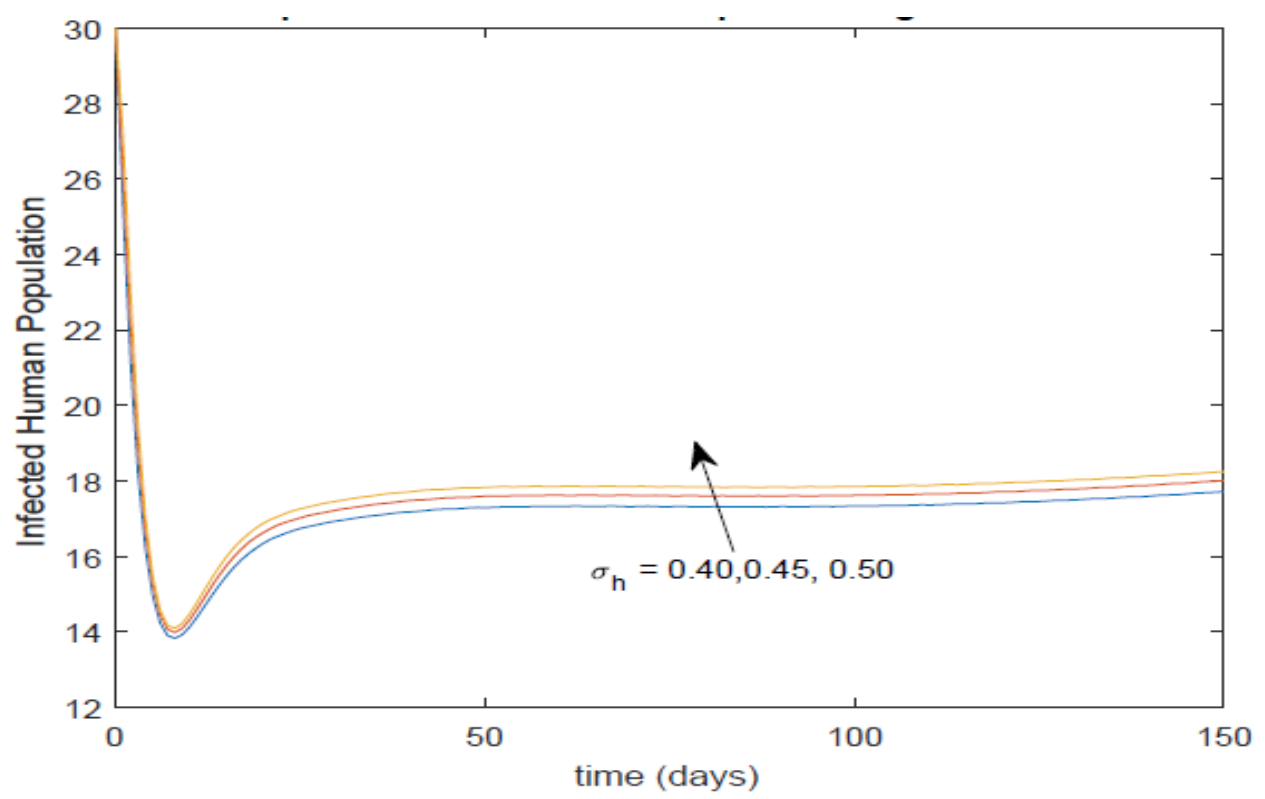

Figure 4.5: Graph of the Infected Human Population against Time varying $\sigma_{h}$

\subsection{Treated Human Population}

Figure 4.6 shows that the treated human population increases rapidly within the first 0 days due to the progression from the infected class into this class. Thereafter, there exist little decrease in the population due to the recovery of the treated humans. 45 member of the treated human population was then maintained for the remaining period of the experiment due to the steady inflow and outflow within the population ascribed to the progression from the infected class and the progression into the recovered class respectively. This result is in sharp agreement with that of $[3]$.

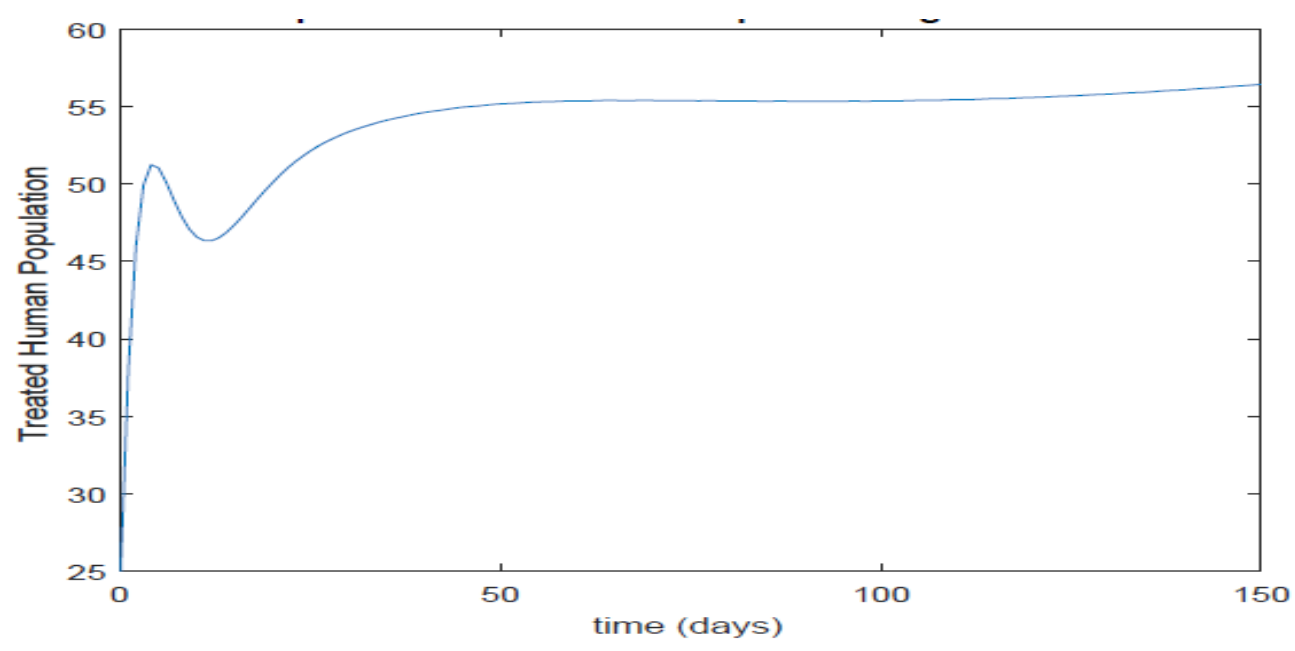

Figure 4.6: Graph of Treated Human Population against Time

\subsection{Recovered Human Population}

Figure 4.7 indicates that the recovered human compartment increases in size rapidly first a during the first 5 days 
of the experiment due to its population by the treated humans who have recovered. Thereafter, there is a little decrease in this compartment ascribed to the progression of the recovered human population into the susceptible class due to loss of immunity. This decrease, however, does not last too long as the treated humans continues to move into the recovered class due to the efficacy of the treatment given. Hence, the recovered human population remains maintained at a stable state after the first 50 days. This result is in agreement with that of [3] but not with that of [4] since they did not consider immunity loss in their model construction. It can be observed that an increase in the rate of recovery of the Carrier class, $\gamma_{1}$ leads to an increase in the population of the recovered class.

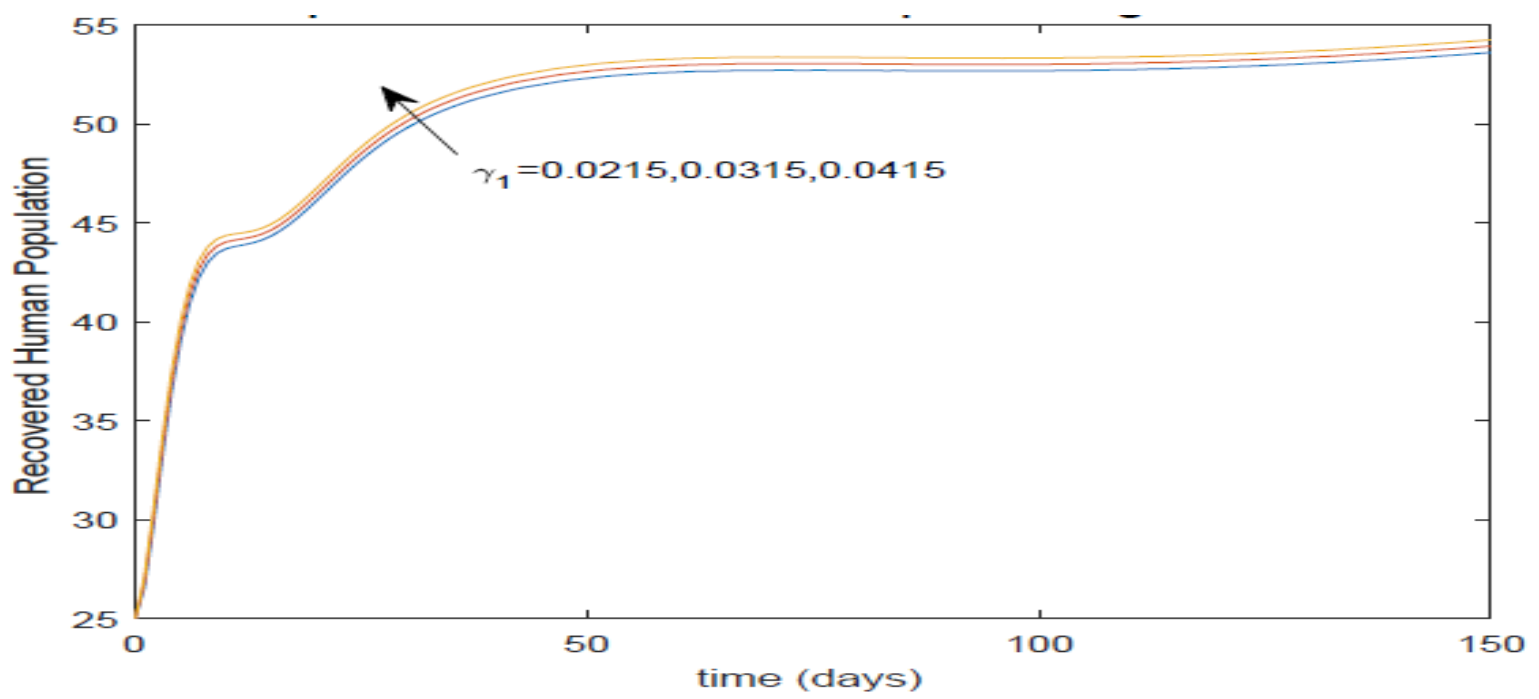

Figure 4.7: Graph of the Recovered Human Population against Time varying $\gamma_{1}$ 4.6 Susceptible Vector Population

From figure 4.8 , the susceptible vector population decreases steadily and continues to decrease for a long period of time due to the progression into the infected vector compartment. After this period, however, the susceptible compartment is maintained at a steady state since it was continually populated either by new births or by recruitments from outside the studied population. However, since there do not exist the use of Rodenticide, decrease in this compartment was only due to natural death or the progression into the infected compartment. This result is in slight disagreement with that of [3] and [4] since they considered Rodenticide factor in their model. The use of Rodenticide, however, shall be introduced as a control parameter during the formulation of the optimal control problem.

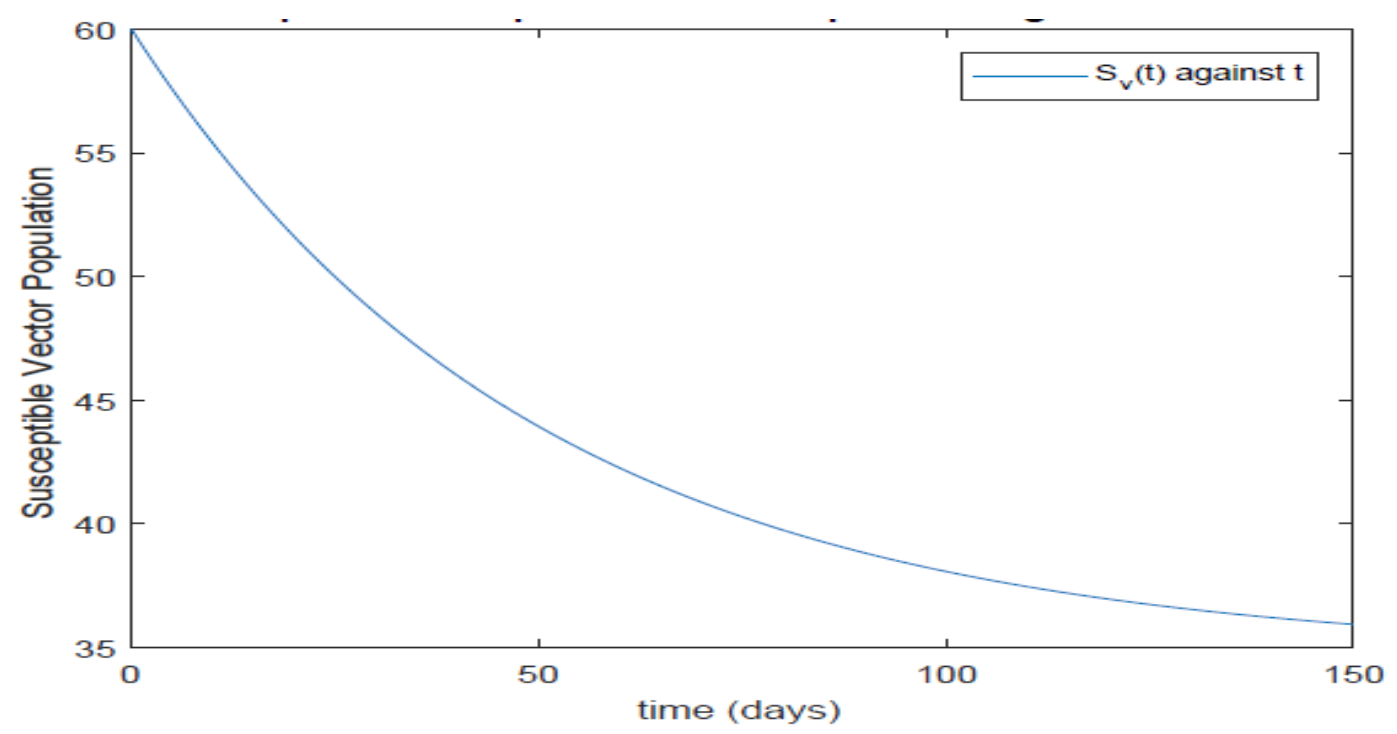

Figure 4.8: Graph of the Susceptible Vector Population against Time 


\subsection{Infected Vector Population}

From figure 4.9, the infected vector population increases rapidly during the first 40 days due to its population by the susceptible vector population and thereafter decreases steadily due to the disease induced death rate and the natural death rate of the vectors. This decrease, however was not progressive since there was no use of Rodenticide nor was their recovery from the infectiousness. This is in contrast with the result of [3], in which there was a rapid decrease in this population due to the application of Rodenticide.

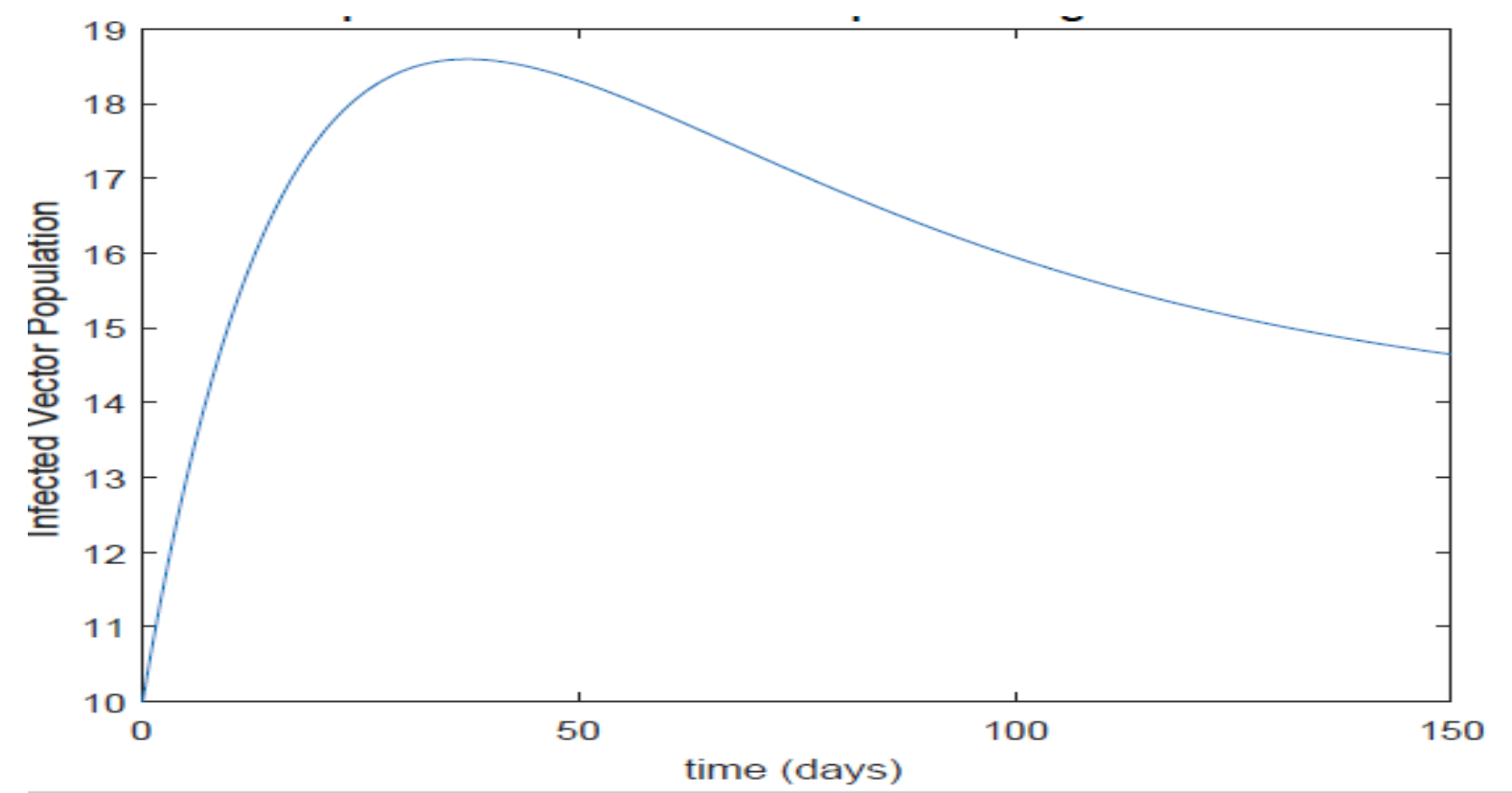

Figure 4.9: Graph of the Infected Vector Population against Time

\section{Conclusion and Recommendations}

The results of the analyses of the developed model indicate that the disease free equilibrium of Lassa fever is stable while the endemic equilibrium point is unstable. These results imply that it is possible to stop the growth and spread of this disease within a studied population provided the assumptions and parameters of the developed model are implemented within such population including the isolation and treatment of the infected class. Similarly, from the simulation of the model, we observe that the carriers and infected class are maintained at a relatively low value by the end of the 150 observed days. However, the infected class is maintained at 17 which is relatively lower than the carrier class which was maintained at 29. This result is because of the availability of treatment for the infected class but not for the carrier class.

Hence, an OCP model which takes into consideration early diagnosis/early treatment of the carrier class alongside other control parameters should be developed and an optimal control application should be made on such model. Also, sensitivity analyses should be performed on the developed model in order to see the specific effect of each parameter of the model on the spread and control of the disease.

\section{References}

[1] Paul Adepoju. Nigeria’s unending war with lassa fever. 627-628, 2019.

[2] S O Adewale, IA Olopade, SO Ajao, GA Adeniran, and OT Oyedemi. Mathematical analysis of lassa fever model with isolation. Asian Journal of Natural \& Applied Sciences, 5(3):47-57, 2016.

[3] A S Afolabi and A R Sobowale. A mathematical model for the control of lassa fever. Transaction of the Nigerian association of mathematical physics, 5(September and November, 2017):279-284, 2017.

[4] J O Akanni and A D Adedipo. Sensitivity analysis of the dynamical transmission and control of lassa fever virus. Asian Research Journal of Mathematics, 9(3):1-11, 2018. 
[5] F O Akinpelu and R Akinwande. Mathematical model for lassa fever and sensitivity analysis. Journal of Scientific and Engineering Research, 5(6):1-9, 2008.

[6] S T Akinyemi, M O Ibrahim, I G Usman, and O Odetunde. Global stability analysis of sir epidemic model with relapse and immunity loss. International Journal of Applied Science and Mathematical Theory, 2(1):1-12, 2016.

[7] M Bawa, S Abdulrahman, O R Jimoh, and N U Adabara. Stability analysis of the disease free equilibrium state for lassa fever disease. Journal of Science, Technology, Mathematics and Education (JOSTMED), 9(2):115$123,2013$.

[8] Carlos Castillo-Chavez and Baojun Song. Dynamical models of tuberculosis and their applications. Mathematical biosciences and engineering, 1(2):361-404, 2004.

[9] CDC. Act against aids, retrieved from https://www.cdc.gov/actagainstaids/basics/transmission.html on 01/09/2018 at 22:05gmt, 2018.

[10] A Dahmane, J Van Griensven, M Van Herp, R Van den Bergh, Y Nzomukunda, J Prior, P Alders, A Jambai, and R Zachariah. Constraints in the diagnosis and treatment of lassa fever and the effect on mortality in hospitalized children and women with obstetric conditions in a rural district hospital in sierra leone. Transactions of the Royal Society of Tropical Medicine and Hygiene, 108(3):126-132, 2014.

[11] A Du-Toit. Lassa fever outbreak in nigeria, retrieved from http://dx.doi.org/10.1038/nrmicro.2018.39 on 01/09/2018 at 12:15gmt. Nature Reviews Microbiology, 16(260), 2018.

[12] S Lukashevich Igor. Pan-lassa fever vaccine for prevention and post-challenge applications. Journal of Vaccines and Vaccination, 8(3):27-33, 2017.

[13] Nicholas N A Kyei, Mark M Abilba, Foster K Kwawu, Prince G Agbenohevi, Joseph H K Bonney, Thomas K Agbemaple, Shirley C Nimo-Paintsil, William Ampofo, Sally-Ann Ohene, and Edward O Nyarko. Imported lassa fever: a report of 2 cases in ghana. BMC infectious diseases, 15(1):217, 2015.

[14] O S Obabiyi and Akindele A Onifade. Mathematical model for lassa fever transmission dynamics with variable human and reservoir population. International Journal of Differential Equations and Applications, 16(1):67-91, 2017.

[15] C O Ogabi, T V Olusa, and M A Madufor. Controlling lassa fever transmission in northern part of edo state, nigeria using sir model. New York Science Journal, 5(12):190-197, 2012.

[16] J O Olayiwola and A S Bakarey. Epidemiological trends of lassa fever outbreaks and insights for future control in nigeria. Int J Trop Dis Heal, 24(4):1-14, 2017.

[17] D Omale and Thomas E. Edibo. Mathematical models for lassa fever transmission with control strategies. Computing, Information Systems, Development Informatics \& Allied Reseach Journal, 6(4):25-31, 2017.

[18] M O Onuorah, N I Akinwande, M O Nasir, and M S Ojo. Sensitivity analysis of lassa fever model. European Centre for Research Training and Developmemt, UK, 4(1):30-49, 2016.

[19] M O Onuorah, M S Ojo, DJ Usman, and A Ademu. Basic reproductive number for the spread and control of lassa fever. International Journal of Mathematics Trends and Technology (IJMTT), 30:1-7, 2016.

[20] O J Peter, O B Akinduko, F A Oguntolu, and C Y Ishola. Mathematical model for the control of infectious disease. Journal of Applied Sciences and Environmental Management, 22(4):447-451, 2018.

[21] Promed. Promed-mail. lassa fever - liberia, retrieved from http://www. promedmail.org/direct.php?id=20180615.5858754 on 01/09/2018 at 12:45GMT, 2018.

[22] PSDS. Pathogen safety data sheets: Infectious substances - lassa virus, retrieved from 
https://www.canada.ca/en/public-health/services/laboratory-biosafety-biosecurity/pathogen-safety-data-sheetsrisk-assessment/lassa-virus.html on 01/09/2018 at 22:20gmt. 2010.

[23] WHO. Emergencies preparedness response, retrieved from http://www.who.int/csr/don/20-april-2018-lassafever-nigeria/en/ on 31/07/2018 at 22:20GMT, 2018.

[24] WHO. Infectious diseases,retrieved from http://www.who.int/topics/infectious_diseases/en/on 17/09/2018 at 13:20GMT, 2018.

[25] WHO. Emergencies preparedness response, retrieved from http://www. who.int/csr/don/14-february-2019lassa-fever-nigeria/en/ on 11/03/2019 at 09:20GMT, 2019. 